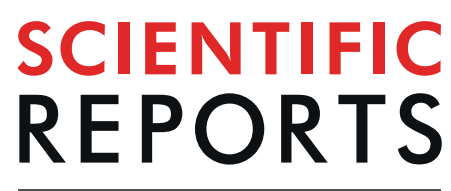

natureresearch

Received: 11 March 2019

Accepted: 25 September 2019

Published online: 15 October 2019

\section{Statin therapy and recurrent venous thromboembolism in the elderly: a prospective cohort study}

\author{
Regula Monika Kronenberg ${ }^{1,6}$, Shanthi Beglinger $\mathbb{1}^{1,2}$, Odile Stalder ${ }^{3}$, Marie Méan ${ }^{4}$, \\ Andreas Limacher ${ }^{3}$, Jürg Hans Beer ${ }^{5}$, Drahomir Aujesky ${ }^{1}$, Nicolas Rodondi ${ }^{1,2}$ \& Martin Feller ${ }^{1,2}$
}

Previous studies reported lower rates of recurrent venous thromboembolism (rVTE) among statin users, but this association could be influenced by concurrent anticoagulation and confounding by statin indication. This study aimed to confirm the beneficial association between statins and rVTE, stratified according to periods with and without anticoagulation, and additionally employ propensity score weighted approach to reduce risk of confounding by indication. The setting was a prospective multicentre cohort study and the outcome was time to first rVTE in statin vs. non-statin users. 980 participants with acute VTE were enrolled (mean age 75.0 years, $47 \%$ women), with median follow-up of 2.5 years. Of $241(24.3 \%)$ statin users, 21 (8.7\%) suffered rVTE vs. 99 (13.4\%) among 739 nonusers. The overall adjusted sub-hazard ratio (aSHR) for rVTE comparing statin users to non-users was $0.72(95 \% \mathrm{Cl} 0.44$ to $1.19, \mathrm{p}=0.20)$. This association was only apparent during periods without anticoagulation (aSHR $0.50,95 \% \mathrm{Cl} 0.27$ to $0.92, p=0.03$; vs. with anticoagulation: aSHR $1.34,95 \% \mathrm{Cl}$ 0.54 to $3.35, \mathrm{p}=0.53$ ). Using propensity scores, the rVTE risk during periods without anticoagulation fell further (aSHR $0.20,95 \% \mathrm{Cl} 0.08$ to $0.49, \mathrm{p}<0.001$ ). In conclusion, statin use is associated with a more pronounced risk reduction for rVTE than previously estimated, but only during periods without anticoagulation.

A recent systematic review and meta-analysis of randomised clinical trials (RCT) and observational studies demonstrated a $15-25 \%$ risk reduction in a first episode of venous thromboembolism (VTE) with statin use ${ }^{1}$. The underlying mechanism of action for this benefit could lie in a reduction of markers of inflammation ${ }^{2}$, resulting in a decreased coagulant state and ultimately a reduced risk for $\mathrm{VTE}^{3,4}$. For persons who have already suffered a VTE, an RCT with statins is lacking. According to a meta-analysis of eight observational studies, the risk of recurrent VTE (rVTE) was 27\% lower under statin therapy, and this association was homogenous across the included studies $\left(\mathrm{I}^{2}=0 \%\right)$, but results were not stratified according to whether or not patients took anticoagulants ${ }^{5}$.

Statin users are more likely to be older ( $>60$ years) and suffer from cardiovascular comorbidities which predispose for VTE, compared to non-users ${ }^{6-8}$. In this instance, a traditional multivariable statistical analysis approach may fall short of balancing comorbidities between statin users and non-users, and the results could be confounded by statin indication ${ }^{9}$. Thus, in the absence of an RCT, it is advised to analyse observational data with a statistical approach that improves balance of baseline comorbidities, and this could be achieved with a propensity score weighted approach ${ }^{9}$.

We aimed to first assess whether statin use was associated with a lower rate of rVTE in a prospective cohort study with standardised assessment of rVTE, second to examine if the magnitude of the association between statin use and rVTE using a traditional multivariate statistical analysis approach was comparable with a propensity score (PS) weighted approach, and third to explore whether markers of inflammation (ultrasensitive C-Reactive Protein [uCRP], fibrinogen) explained the effect of statins.

\footnotetext{
${ }^{1}$ Department of General Internal Medicine, Inselspital, Bern University Hospital, University of Bern, Bern, Switzerland. ${ }^{2}$ Institute of Primary Health Care (BIHAM), University of Bern, Bern, Switzerland. ${ }^{3}$ CTU Bern, and Institute of Social and Preventive Medicine (ISPM), University of Bern, Bern, Switzerland. 'Department of Medicine, Division of Internal Medicine, Lausanne University Hospital, Lausanne, Switzerland. ${ }^{5}$ Department of Internal Medicine, Cantonal Hospital of Baden, Baden, Switzerland. ${ }^{6}$ Department of Internal Medicine, Cantonal Hospital of Luzern, Wolhusen, Switzerland. Correspondence and requests for materials should be addressed to S.B. (email: shanthi.beglinger@extern.insel.ch)
} 


\section{Methods}

We based our study report on the guidelines provided in the Strengthening the Reporting of Observational studies in Epidemiology (STROBE) statement ${ }^{10}$.

Study design, setting, and participants. This study was conducted between September $2^{\text {nd }}, 2009$ and December $6^{\text {th }}$, 2013. It was part of the Swiss Cohort of Elderly Patients with VTE (SWITCO65+) prospective multicentre cohort study, which assessed long-term medical outcomes in elderly patients with acute VTE. The study population was recruited from all five university (Basel, Bern, Geneva, Lausanne and Zurich) and four high-volume non-university hospitals (cantonal hospitals of Baden, Frauenfeld, Luzern and St. Gallen) in Switzerland. The Ethics Committee at each of the nine participating centres approved the study. All research in this observational study was performed in accordance with relevant ethics and research guidelines/regulations. Informed consent to participate in the SWITCO65 + study was obtained for all enrolled adults. Participants aged 65 years or older, with an acute, objectively confirmed symptomatic deep vein thrombosis (DVT) or symptomatic pulmonary embolism (PE), were prospectively recruited in the inpatient and outpatient settings of all study sites, and followed-up until December 6th, 2013 (participants were censored earlier in case of death or rVTE $)^{11}$. Symptomatic PE was defined as a positive spiral computed tomography or pulmonary angiography, a high probability ventilation-perfusion scan, or proximal DVT confirmed by compression ultrasonography or contrast venography, in participants with acute chest pain, new or worsening dyspnoea, haemoptysis, or syncope. Those with catheter-related thrombosis, insufficient German-speaking or French-speaking ability, no follow-up possibility (e.g. terminal illness), an inability to provide informed consent (e.g. severe dementia), or previous enrolment in the cohort, were excluded. The SWITCO65+ study was funded by grants from the Swiss National Science Foundation (grants 33CSCO-122659/139470).

Definition of exposures, outcomes, and potential confounders. The main exposure was statin use at study entry (statin user vs. non-user). The main outcome was the time to the first recurrence of a symptomatic, objectively confirmed VTE (rVTE) during the follow-up period, defined as PE or DVT (proximal and/or distal) based on previously published criteria ${ }^{11-13}$. Follow-up started directly after diagnosis of index VTE. The first follow-up took place after three months. During follow-up, study nurses interviewed participants (or respectively general practitioners in case of death, or relatives in case of no information [if participant died at home]) about the date and type of clinical events (rVTE; death). These interviews were implemented as one telephone call and two surveillance face-to-face evaluations during the first year of study participation. Following this, semi-annual contact was initiated which alternated between face-to-face evaluations (clinic visits or home visits in housebound participants), telephone calls, and periodic review of the participant's hospital chart. If a clinical event did occur, the participant's medical chart was reviewed, and interviews were held with the participant's primary care physicians and family members.

In addition, information about the following variables that were relevant for the statistical analyses was collected: age (analysed as a continuous variable), smoking history (never, past, current), polypharmacy ( $<5$ drugs vs. $\geq 5$ drugs), prior VTE (i.e. a VTE that occurred before the index VTE), transient provoked VTE (surgery during the last 3 months, immobilisation during the last 3 months, oestrogen therapy during the last 3 months), cardiovascular disease (coronary heart disease, peripheral artery disease, cerebrovascular disease [stroke, TIA]), arterial hypertension, diabetes mellitus, body-mass index (BMI) $\geq 25$ [yes vs. no], fibrinogen and uCRP (uCRP was measured at University Hospital Zurich in serum aliquots using a latex-enhanced immunoturbidimetric assay on a cobas c502 autoanalyser [Roche Diagnostics, Mannheim, Germany] with assay characteristics as reported by the manufacturer). A committee of three blinded clinical experts adjudicated all outcomes, and final classifications were made based on the full consensus of this committee ${ }^{11}$.

Statistical analyses. We compared baseline characteristics among statin users and non-users with the chi-squared test and the non-parametric Wilcoxon rank-sum test as appropriate. rVTE rates were displayed for statin users and non-users, as well as stratified into periods with and without anticoagulation. We examined the overall association between statin use and the time to a first rVTE using competing risk regression, accounting for non-PE-related death as a competing event. The method yields subhazard ratios (SHR) with corresponding $95 \%$ CIs and p-values for the failure event of primary interest. In the traditional statistical analysis approach, we adjusted for established risk factors for VTE: age, sex, symptomatic PE, prior VTE, transient provoked VTE, cardiovascular disease (i.e. coronary heart, peripheral arterial or cerebrovascular [stroke, TIA] disease), active cancer, and periods of anticoagulation as a time-varying covariate. In a sensitivity analysis, we additionally adjusted for Aspirin, other antiplatelet therapy, hypercholesterolaemia and diabetes after observing that these characteristics were not evenly distributed among statin users and non-users. In another sensitivity analysis, we stratified the results into periods with and without anticoagulation. Moreover, since the study population included both provoked and unprovoked index VTE, we also performed subgroup analysis to examine whether the association between statin use and rVTE rates was similar between these two groups. Furthermore, we assessed fibrinogen and uCRP as potential inflammatory mediators, additionally correcting models for their log-transformed values. Beforehand, we performed multiple imputation for missing values of fibrinogen and uCRP.

In the PS weighted analysis approach, we calculated the propensity to receive a statin in a logistic model with statin use as dependent and all the variables that were used for adjustment in the main analysis as independent variables. The advantage of the PS approach was the ability to not only better balance the two groups (statin users and non-users) in terms of baseline characteristics, but to also account for more potential confounders if rVTE events were few. Therefore, in the PS approach we additionally adjusted for hypertension, polypharmacy, smoking status, and BMI. Missing values for BMI and smoking status were assumed to be normal (i.e. BMI as $<25$ [n=4], 


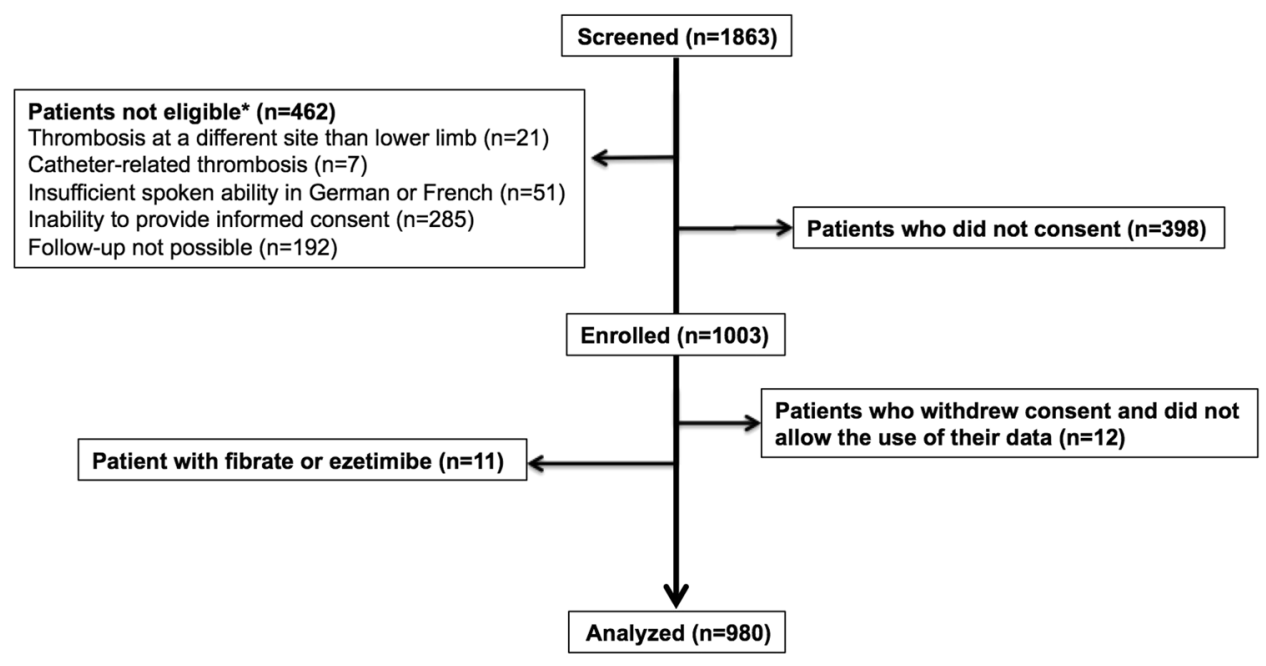

Figure 1. Flow-chart enrolment process.

smoking status as never [n=3]). For completion, we compared the results from the PS approach with a sensitivity analysis from the traditional statistical approach, which adjusted for all the same variables as in the PS analysis.

We trimmed the PS values using the $2.5^{\text {th }}$ percentile of the PS in the statin users as the lower cut-off and the $97.5^{\text {th }}$ percentile of the PS in the non-users as the upper cut-off. Observations in both groups that had PS values beyond these two cut-offs were dropped ${ }^{14}$. We then calculated the inverse probability of treatment weights (IPTW) for the remaining participants. The competing risk model was weighted according to the IPTW and additionally adjusted for periods of anticoagulation as a time-varying covariate. Again, we stratified the results into periods with and without anticoagulation in sensitivity analyses, and also assessed fibrinogen and uCRP as potential inflammatory mediators, additionally correcting models for their log-transformed values. We used Stata 15 for all analyses (Stata Corporation, College Station, Texas).

\section{Essentials.}

- Statin users have lower rates of recurrent venous thromboembolism (rVTE), but the magnitude of this association is uncertain and could be influenced by concurrent anticoagulation.

- While statin therapy was not associated with reduced risk of rVTE during periods of anticoagulation, we observed a 50\% reduced risk of rVTE with statin therapy during periods without anticoagulation.

- Using a propensity score weighted approach, statins were associated with an $80 \%$ reduced risk of rVTE during periods without anticoagulation.

\section{Results}

Participants. Between September 2009 and March 2012, 1,003 participants with VTE were enrolled and followed until December $2013^{11}$. For this analysis, we excluded 11 participants with fibrate or ezetimibe treatment (Fig. 1), because these drugs are possibly associated with a higher risk of VTE ${ }^{15,16}$. We further excluded four participants who withdrew informed consent within one day, and eight participants who denied use of their data.

The 980 included participants had a mean age of 75.0 years, $52.7 \%$ were men, $99.8 \%$ were Caucasian. The majority of participants had comorbidities, such as active cancer, chronic lung disease, or heart failure (Table 1). Overall, $69.3 \%$ of participants had PE at baseline, with or without DVT. Most participants (81.2\%) were treated in hospital, and low-molecular-weight heparin was administered to $48.6 \%$ of participants as initial antithrombotic treatment.

$241(24.3 \%)$ participants reported statin use compared to 739 non-users. Among the statin users, 103 took atorvastatin, 73 simvastatin, 49 pravastatin, 12 rosuvastatin, and 4 fluvastatin. Statin users and non-users had a similar age and sex distribution, but statin users were more overweight (BMI $\geq 25 \mathrm{~kg} / \mathrm{m}^{2}: 72 \% \mathrm{vs.} 62 \%$ ) and were generally in a worse medical condition suffering from more comorbidities, with a higher prevalence of diabetes mellitus ( $25 \%$ vs. $12 \%$ ), arterial hypertension ( $79 \%$ vs. $59 \%)$, chronic or acute heart failure (19\% vs. $9 \%$ ), coronary heart disease ( $42 \%$ vs. $9 \%)$, peripheral arterial disease (12\% vs. $5 \%)$, cerebrovascular disease ( 21 vs. $5 \%)$, past major bleeds ( 15 vs. $9 \%$ ), and polypharmacy ( 80 vs. $41 \%)$.

Statins and rVTE. After a mean follow-up of 27.5 months (median 29.6 months), 21 of the 241 statin users (8.7\%) experienced a rVTE compared to 99 of the 739 non-users (13.4\%). rVTE incidence rates (IR) were 3.8 per 100 patient-years $(95 \%$ CI 2.5 to 5.9$)$ among statin users vs. 6.3 per 100 patient-years $(95 \%$ CI 5.2 to $7.7, \mathrm{p}=0.036)$ among non-users. During periods with anticoagulation, rVTE IR were 2.5 per 100 patient-years $(95 \%$ CI 1.3 to 4.8) among statin users vs. 2.3 per 100 patient-years (95\%CI 1.5 to 3.5) among non-users. During periods without anticoagulation, rVTE IR were 6.4 per 100 patient-years (95\%CI 3.6 to 11.3 ) among statin users vs. 12.6 per 100 patient-years (95\%CI 10.1 to 15.8 ) among non-users. Overall, statin users had a $38 \%$ reduced risk of rVTE 


\begin{tabular}{|c|c|c|c|}
\hline \multirow[b]{3}{*}{ Total N } & \multirow{2}{*}{\begin{tabular}{|l|} 
Statin \\
$\mathbf{n}(\%)$ or median (IQ-range) \\
\end{tabular}} & \multirow[b]{2}{*}{ No statin } & \multirow[b]{3}{*}{ p-value } \\
\hline & & & \\
\hline & $\mathrm{N}=\mathbf{2 4 1}$ & $\mathrm{N}=739$ & \\
\hline Age [years] & $75.0(70.0 ; 81.0)$ & $75.0(69.0 ; 81.0)$ & 0.63 \\
\hline Females & $107(44 \%)$ & $351(47 \%)$ & 0.40 \\
\hline Smoking status & & & 0.77 \\
\hline current smoker & $18(7 \%)$ & $58(8 \%)$ & \\
\hline past smoker & $103(43 \%)$ & $295(40 \%)$ & \\
\hline never smoker & $120(50 \%)$ & $383(52 \%)$ & \\
\hline Family history of pulmonary embolism/deep vein thrombosis & $37(15 \%)$ & $125(17 \%)$ & 0.57 \\
\hline Known thrombophilia & $2(1 \%)$ & $11(1 \%)$ & 0.44 \\
\hline Diabetes mellitus & $61(25 \%)$ & $91(12 \%)$ & $<0.01$ \\
\hline Hypercholesterolaemia & $206(85 \%)$ & $87(12 \%)$ & $<0.01$ \\
\hline Coronary heart disease & $101(42 \%)$ & $68(9 \%)$ & $<0.01$ \\
\hline Peripheral arterial disease & $29(12 \%)$ & $40(5 \%)$ & $<0.01$ \\
\hline Cerebrovascular disease (stroke/ transient ischaemic attack) & $50(21 \%)$ & $40(5 \%)$ & $<0.01$ \\
\hline \multicolumn{4}{|l|}{ Cardiovascular disease (coronary/peripheral/ } \\
\hline cerebrovascular) & $180(75 \%)$ & $148(20 \%)$ & $<0.01$ \\
\hline Arterial hypertension & & & $<0.01$ \\
\hline no & $49(20 \%)$ & $301(41 \%)$ & \\
\hline yes (treated) & $189(78 \%)$ & $416(56 \%)$ & \\
\hline yes (untreated) & $3(1 \%)$ & $22(3 \%)$ & \\
\hline Chronic lung disease & $34(14 \%)$ & $102(14 \%)$ & 0.91 \\
\hline Body-mass index $\left[\mathrm{kg} / \mathrm{m}^{2}\right]$ & $27.7(24.6 ; 30.8)$ & $26.4(23.8 ; 29.7)$ & $<0.01$ \\
\hline Body-mass index $\geq 25$ & $174(72 \%)$ & $463(63 \%)$ & $<0.01$ \\
\hline Symptomatic pulmonary embolism & $186(77 \%)$ & $491(66 \%)$ & $<0.01$ \\
\hline Prior venous thromboembolism ${ }^{\mathrm{b}}$ & $75(31 \%)$ & $203(27 \%)$ & 0.28 \\
\hline Major surgery during the last 3 months & $44(18 \%)$ & $102(14 \%)$ & 0.09 \\
\hline Current oestrogen therapy during the last 3 months & $4(2 \%)$ & $28(4 \%)$ & 0.11 \\
\hline Immobilisation during the last 3 months & $57(24 \%)$ & $159(22 \%)$ & 0.49 \\
\hline Provoked venous thromboembolism & $75(31 \%)$ & $219(30 \%)$ & 0.66 \\
\hline Active cancer & $38(16 \%)$ & $138(19 \%)$ & 0.31 \\
\hline History of major bleeding & $37(15 \%)$ & $63(9 \%)$ & $<0.01$ \\
\hline Chronic renal disease & $67(28 \%)$ & $117(16 \%)$ & $<0.01$ \\
\hline Polypharmacy & $193(80 \%)$ & $304(41 \%)$ & $<0.01$ \\
\hline Anticoagulation prior to venous thromboembolism & $19(8 \%)$ & $32(4 \%)$ & 0.03 \\
\hline Initial parenteral anticoagulation & $232(96 \%)$ & $714(97 \%)$ & 0.80 \\
\hline Type of initial parenteral anticoagulation & & & 0.02 \\
\hline Low molecular weight heparin & $94(39 \%)$ & $366(50 \%)$ & \\
\hline Unfractionated heparin & $97(40 \%)$ & $233(32 \%)$ & \\
\hline Fondaparinux & $40(17 \%)$ & $115(16 \%)$ & \\
\hline Danaparoid & $1(0 \%)$ & $0(0 \%)$ & \\
\hline None & $9(4 \%)$ & $25(3 \%)$ & \\
\hline Initial vitamin $\mathrm{K}$ antagonist therapy & $211(88 \%)$ & $640(87 \%)$ & 0.705 \\
\hline Concomitant antiplatelet therapy & $149(62 \%)$ & $166(22 \%)$ & $<0.001$ \\
\hline Aspirin & $127(53 \%)$ & $154(21 \%)$ & $<0.001$ \\
\hline Duration of initial anticoagulation [months] & $11.1(5.2 ; 28.7)$ & $7.1(3.7 ; 23.7)$ & 0.012 \\
\hline Fibrinogen at the time of venous thromboembolism $[\mathrm{g} / \mathrm{L}]$ & $4.8(3.7 ; 5.8)$ & $4.5(3.6 ; 5.6)$ & 0.102 \\
\hline $\begin{array}{l}\text { Ultrasensitive C-Reactive Protein at the time of venous } \\
\text { thromboembolism }[\mathrm{mg} / \mathrm{L}]\end{array}$ & $22.2(6.1 ; 63.6)$ & $23.6(8.5 ; 62.1)$ & 0.260 \\
\hline
\end{tabular}

Table 1. Baseline characteristics of statin users and non-users ${ }^{\mathrm{a}} \cdot{ }^{\mathrm{a}}$ Missing: $3(0 \%)$ for smoking status, 8 ( $\left.1 \%\right)$ for family history of venous thromboembolism, 18 (2\%) for systolic blood pressure, 4 ( $0 \%)$ for body-mass index, $1(0 \%)$ for oestrogen therapy, 1 (0\%) for history of major bleeding, $125(13 \%)$ for fibrinogen, $124(13 \%)$ for

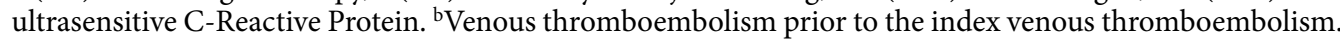

during follow-up compared to non-users (crude SHR 0.62, 95\%CI 0.39 to 1.00, $\mathrm{p}=0.05$ ). After adjusting for age, sex, symptomatic PE, prior VTE, transient provoked VTE, cardiovascular disease, active cancer, and periods of anticoagulation as a time-varying covariate, the effect estimate was slightly attenuated (adjusted [a]SHR $0.72,95 \%$ CI 0.44 to $1.19, \mathrm{p}=0.20$; Table 2 ). In the sensitivity analysis additionally adjusting for Aspirin, other 


\begin{tabular}{|l|l|l|l|l|l|}
\hline $\begin{array}{l}\text { Number of events/ } \\
\text { Number of patients }\end{array}$ & Analysis & $\begin{array}{l}\text { Crude subhazard } \\
\text { ratio (95\%-CI) }\end{array}$ & p-value & $\begin{array}{l}\text { Adjusted subhazard } \\
\text { ratio (95\%-CI) }\end{array}$ & p-value \\
\hline & Traditional statistical analysis approach & & & & \\
\hline $120 / 980$ & Overall & $0.62(0.39$ to 1.00$)$ & 0.049 & $0.72(0.44 \text { to } 1.19)^{\mathrm{a}}$ & 0.197 \\
\hline $31 / 972$ & Periods with AC & $1.16(0.54$ to 2.49$)$ & 0.707 & $1.34(0.54 \text { to } 3.35)^{\mathrm{a}}$ & 0.525 \\
\hline $89 / 595$ & Periods without AC & $0.52(0.28$ to 0.95$)$ & 0.034 & $0.50(0.27 \text { to } 0.92)^{\mathrm{a}}$ & 0.027 \\
\hline & $\begin{array}{l}\text { Sensitivity analysis including same variables } \\
\text { as the propensity score approach }\end{array}$ & & & $0.60(0.36 \text { to } 1.01)^{\mathrm{b}}$ & 0.056 \\
\hline & Including markers of inflammation & & & & \\
\hline $120 / 980$ & Fibrinogen & & & $0.75(0.45 \text { to } 1.23)^{\mathrm{c}}$ & 0.250 \\
\hline $120 / 980$ & ultrasensitive CRP & & & $0.72(0.43 \text { to } 1.19)^{\mathrm{c}}$ & 0.197 \\
\hline & Propensity score weighted approach & & \\
\hline $99 / 792$ & Overall & & & & \\
\hline $28 / 788$ & Periods with AC & $1.34(0.60$ to 3.01$)$ & 0.481 & $1.17(0.47 \text { to } 2.90)^{\mathrm{e}}$ & 0.740 \\
\hline $71 / 477$ & Periods without AC & $0.38(0.18$ to 0.84$)$ & 0.016 & $0.20(0.08 \text { to } 0.49)^{\mathrm{e}}$ & $<0.001$ \\
\hline & Including markers of inflammation & & & & \\
\hline $99 / 792$ & Fibrinogen & & & $0.43(0.22 \text { to } 0.84)^{\mathrm{c}}$ & 0.014 \\
\hline $99 / 792$ & ultrasensitive CRP & & & $0.41(0.21 \text { to } 0.80)^{\mathrm{c}}$ & 0.009 \\
\hline
\end{tabular}

Table 2. Statin therapy and $\mathrm{rVTE} . \mathrm{AC}=$ anticoagulation, $\mathrm{CRP}=\mathrm{C}$-Reactive Protein. ${ }^{\mathrm{a}}$ Adjusted for age, gender, symptomatic pulmonary embolism, prior venous thromboembolism, provoked venous thromboembolism, cardiovascular disease (i.e. coronary heart, peripheral arterial or cerebrovascular [stroke, transient ischaemic attack] disease), active cancer, and periods of anticoagulation as a time-varying covariate. ${ }^{\text {'Sensitivity }}$ analysis: further adjustment for the same variables as used in the propensity score approach. Adjusted to include age, gender, symptomatic pulmonary embolism, prior venous thromboembolism, provoked venous thromboembolism, cardiovascular disease (i.e. coronary heart, peripheral arterial or cerebrovascular [stroke, transient ischaemic attack] disease), active cancer, periods of anticoagulation as a time-varying covariate, and additionally hypertension, polypharmacy, smoking (never/past/current), body-mass index ( $\geq 25)$. ${ }^{c}$ Two separate additional adjustment variables: log-Fibrinogen and log-ultrasensitive C-Reactive Protein (as potential explanatory variables of the association between statins and rVTE). Multiple imputation for missing values of fibrinogen and ultrasensitive C-Reactive Protein was performed. ${ }^{\mathrm{d}}$ Variables used to calculate the propensity score: age, gender, symptomatic pulmonary embolism, prior venous thromboembolism, provoked venous thromboembolism, cardiovascular disease (i.e. coronary heart, peripheral arterial or cerebrovascular [stroke, transient ischaemic attack] disease), active cancer, hypertension, polypharmacy, smoking (never/past/current),

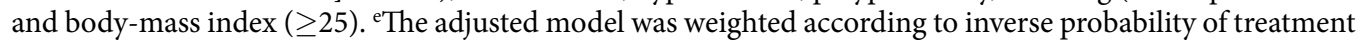
weights (IPTW) and additionally adjusted for periods of anticoagulation as a time-varying covariate.

antiplatelet therapy, hypercholesterolaemia and diabetes, statins were more strongly associated with $\mathrm{rVTE}$ (aSHR $0.56,95 \%$ CI 0.32 to $0.99, \mathrm{p}=0.05$ ). When only time-periods with anticoagulation were considered, there was no association between statin use and $\operatorname{rVTE}$ (aSHR $1.34,95 \% \mathrm{CI} 0.54$ to $3.35, \mathrm{p}=0.53$ ). In contrast, when only time-periods without anticoagulation were considered, statin use was more strongly associated with rVTE (aSHR $0.50,95 \%$ CI 0.27 to $0.92, p=0.03$ ). Upon subgroup analysis, the incidence rates of rVTE were slightly lower in patients with a provoked index VTE (IR 4.6 per 100 patient-years, 95\% CI 3.2 to 6.6) compared to unprovoked index VTE patients (IR 6.1 per 100 patient-years, 95\%CI 5.0 to 7.5). Nevertheless, reduction in rVTE risk with statin use compared to non-use was still similar among the two subgroups (provoked index VTE - aSHR 0.71, $95 \%$ CI 0.24 to $2.15, \mathrm{p}=0.55$; unprovoked VTE - aSHR $0.68,95 \%$ CI 0.39 to $1.20, \mathrm{p}=0.19$ ); confirming that there was no observable subgroup effect ( $\mathrm{p}$ for interaction 0.93 ). Neither fibrinogen nor uCRP had an impact on the results (aSHR including fibrinogen $0.75,95 \% \mathrm{CI} 0.45$ to $1.23, \mathrm{p}=0.25$; aSHR including uCRP $0.72,95 \% \mathrm{CI} 0.43$ to $1.19, \mathrm{p}=0.20$; Table 2).

In the PS weighted analysis (based on 792 participants with $99 \mathrm{rVTEs}$ ), we observed a more pronounced rVTE risk reduction of $58 \%$ among statin users compared to non-users (aSHR $0.42,95 \%$ CI 0.21 to $0.81, \mathrm{p}=0.01$ ). Again, the association became stronger during periods without anticoagulation (aSHR 0.20, 95\%CI 0.08 to $0.49, \mathrm{p}<0.001$ ), whereas it was not observed during periods of anticoagulation (aSHR 1.17, 95\%CI 0.47 to 2.90, $\mathrm{p}=0.74$ ). Further, neither fibrinogen nor uCRP had an impact on the results (aSHR including fibrinogen 0.43, 95\%CI 0.22 to $0.84, \mathrm{p}=0.01$; aSHR including uCRP $0.41,95 \% \mathrm{CI} 0.21$ to $0.80, \mathrm{p}=0.01$; Table 2 ). In a sensitivity analysis using the traditional statistical analysis approach, but adjusting for all variables that were used in the PS analysis, the association between statin use and rVTE was slightly stronger compared to the original multivariable approach (aSHR 0.60, 95\%CI 0.36 to $1.01, \mathrm{p}=0.06$ vs. aSHR 0.72, 95\% CI 0.44 to $1.19, \mathrm{p}=0.20$ ).

\section{Discussion}

In this population-based prospective cohort study, we found a lower rate of rVTE among statin users compared to non-users, but this beneficial association was only apparent during periods without anticoagulation, and stronger in the PS weighted approach than in the traditional statistical analysis approach. Furthermore, the potentially beneficial effect of statins was not explained by markers of inflammation such as fibrinogen or uCRP. 
Our results from the traditional statistical analysis approach were very similar to a recent meta-analysis of eight observational studies ${ }^{5}$, indicating an almost $30 \%$ risk reduction in rVTE with statin therapy. However, our results suggest that statins may only reduce the risk for rVTE in the absence of anticoagulation. Therefore, they might be a second best option to prevent rVTE when anticoagulation is contraindicated. Moreover, under the PS weighted approach, the risk reduction became even more substantial (i.e. $80 \%$ during periods without anticoagulation). There are arguments supporting the hypothesis that the traditional statistical analysis approach underestimates the true effect of statins in observational studies: statin users have more comorbidities and are sicker than non-users ${ }^{6,7}$, which is also observed in our own study population's baseline characteristics (Table 1), and multimorbid persons have a higher risk of $\mathrm{VTE}^{8}$. Thus, the potentially beneficial effect of statins could be partly concealed by comparing multimorbid statin users with healthier non-users in observational studies using a traditional multivariable statistical analysis approach. In contrast, in the PS weighted approach, participants with a PS value below the $2.5^{\text {th }}$ percentile and above the $97.5^{\text {th }}$ percentile were discarded, as at these extremes of range there was no comparable participant in the other group with similar comorbidities/risk profile, thus making the two groups (statin users vs. non-users) as similar as possible ${ }^{14}$.

Our study has several limitations. First, the study population was recruited consecutively from most large-volume hospitals in Switzerland. As a result, it is uncertain if the results also apply to patients with potentially less severe (r)VTE who were treated in smaller hospitals or by general practitioners. Second, almost all study participants were white, so our results are only generalisable to a Caucasian population. Third, we tested if the potential beneficial effect of statins was explained by a reduction in inflammation - we observed no such association. Yet, we only considered fibrinogen and uCRP. Both markers have been suggested by previous studies to be affected by statin therapy ${ }^{17,18}$. It is possible that statins reduce the risk of (r)VTE via other inflammatory markers for which we had no information available or via direct impact on endothelial function ${ }^{17,19}$. In fact, newly published data suggests that the protective effect of statins in decreasing rVTE rates may not be down to a reduction in inflammation, but rather due to a reduction in coagulation factors ${ }^{20}$. Fourth, our study did not have sufficient power to analyse different statin agents separately, and it is possible that the risk reduction seen in (r) VTE is statin-specific and therefore, cannot be attributed to a class-effect ${ }^{21,22}$. Fifth, all participants were 65 years or older. Therefore, it is uncertain whether our results also apply to a younger population. Sixth, whether or not a participant used statins was only assessed at baseline. Therefore, our exposure assessment (i.e. statin use) might be inaccurate in cases where participants started or stopped statin therapy during follow-up. However, as this inaccuracy is not related to the outcome of interest (rVTE), results may at worst be biased towards the null (i.e. underestimate the association between statin use and rVTE).

Our study has several strengths. First, it highlights that statistical analysis approaches accounting for confounding by indication (i.e. the PS weighted approach) can produce different results from traditional multivariable analyses of observational data. Analysing observational data with both a traditional multivariable analysis and a PS weighted analysis, comparing the findings and attempting to explain differences, can result in new insights. Furthermore, it makes optimal use of already existing data. Second, our study included elderly patients with VTE (mean age 75 years), a population that is often underrepresented or excluded from clinical trials. Third, the cohort had a small dropout rate of $4.8 \%$ during follow-up ${ }^{11}$, and the data collection was near complete with less than $5 \%$ missing values. Fourth, rVTE was adjudicated by a blinded expert committee using clearly defined previously published objective criteria ${ }^{11-13}$, reducing the risk of detection bias. Finally, both inpatients and outpatients with an index VTE were included in a nationwide multicentre setting.

\section{Conclusion}

Statin use was associated with a more pronounced risk reduction for rVTE than previously estimated, but only without concurrent anticoagulation. An RCT would be needed to substantiate the potential benefits of statins for prevention of rVTE during periods when anticoagulation is not indicated or feasible.

\section{Data Availability}

All necessary data that support the findings of this study are included within this published article. Any further supplemental data is available from the authors upon request.

\section{References}

1. Kunutsor, S. K., Seidu, S. \& Khunti, K. Statins and primary prevention of venous thromboembolism: a systematic review and metaanalysis. The Lancet. Haematology 4, e83-e93, https://doi.org/10.1016/S2352-3026(16)30184-3 (2017).

2. Arslan, F., Pasterkamp, G. \& de Kleijn, D. P. Unraveling pleiotropic effects of statins: bit by bit, a slow case with perspective. Circulation research 103, 334-336, https://doi.org/10.1161/CIRCRESAHA.108.182220 (2008).

3. Albert, M. A., Danielson, E., Rifai, N., Ridker, P. M. \& Investigators, P. Effect of statin therapy on C-reactive protein levels: the pravastatin inflammation/CRP evaluation (PRINCE): a randomized trial and cohort study. JAMA 286, 64-70 (2001).

4. van Aken, B. E., den Heijer, M., Bos, G. M., van Deventer, S. J. \& Reitsma, P. H. Recurrent venous thrombosis and markers of inflammation. Thromb Haemost 83, 536-539 (2000).

5. Kunutsor, S. K., Seidu, S. \& Khunti, K. Statins and secondary prevention of venous thromboembolism: pooled analysis of published observational cohort studies. Eur Heart J 38, 1608-1612, https://doi.org/10.1093/eurheartj/ehx107 (2017).

6. Yang, C. C., Jick, S. S. \& Testa, M. A. Who receives lipid-lowering drugs: the effects of comorbidities and patient characteristics on treatment initiation. British journal of clinical pharmacology 55, 288-298 (2003).

7. Lipworth, L. et al. A prospective study of statin use and mortality among 67,385 blacks and whites in the Southeastern United States. Clinical epidemiology 6, 15-25, https://doi.org/10.2147/clep.s53492 (2014).

8. Geerts, W. H. et al. Prevention of venous thromboembolism. Chest 119, 132S-175S (2001).

9. Johnson, S. R., Tomlinson, G. A., Hawker, G. A., Granton, J. T. \& Feldman, B. M. Propensity Score Methods for Bias Reduction in Observational Studies of Treatment Effect. Rheumatic diseases clinics of North America 44, 203-213, https://doi.org/10.1016/j. rdc.2018.01.002 (2018).

10. von Elm, E. et al. The Strengthening the Reporting of Observational Studies in Epidemiology (STROBE) statement: guidelines for reporting observational studies. Lancet (London, England) 370, 1453-1457, https://doi.org/10.1016/S0140-6736(07)61602-X (2007). 
11. Mean, M. et al. The Swiss cohort of elderly patients with venous thromboembolism (SWITCO65+): rationale and methodology. J Thromb Thrombolysis 36, 475-483, https://doi.org/10.1007/s11239-013-0875-2 (2013).

12. Buller, H. R. et al. Subcutaneous fondaparinux versus intravenous unfractionated heparin in the initial treatment of pulmonary embolism. N Engl J Med 349, 1695-1702, https://doi.org/10.1056/NEJMoa035451 (2003).

13. Columbus, I. et al. Low-molecular-weight heparin in the treatment of patients with venous thromboembolism. N Engl J Med 337, 657-662, https://doi.org/10.1056/NEJM199709043371001 (1997).

14. Sturmer, T., Rothman, K. J., Avorn, J. \& Glynn, R. J. Treatment effects in the presence of unmeasured confounding: dealing with observations in the tails of the propensity score distribution-a simulation study. Am J Epidemiol 172, 843-854, https://doi. org/10.1093/aje/kwq198 (2010).

15. Delluc, A. et al. Lipid lowering drugs and the risk of recurrent venous thromboembolism. Thromb Res 130, 859-863, https://doi. org/10.1016/j.thromres.2012.08.296 (2012).

16. Squizzato, A. et al. Statins, fibrates, and venous thromboembolism: a meta-analysis. Eur Heart J 31, 1248-1256, https://doi. org/10.1093/eurheartj/ehp556 (2010).

17. Undas, A., Brummel-Ziedins, K. E. \& Mann, K. G. Statins and blood coagulation. Arterioscler Thromb Vasc Biol 25, 287-294, https:// doi.org/10.1161/01.ATV.0000151647.14923.ec (2005).

18. Bickel, C. et al. Relation of markers of inflammation (C-reactive protein, fibrinogen, von Willebrand factor, and leukocyte count) and statin therapy to long-term mortality in patients with angiographically proven coronary artery disease. Am J Cardiol 89, 901-908 (2002).

19. Kim, Y. S. et al. Rosuvastatin suppresses the inflammatory responses through inhibition of c-Jun N-terminal kinase and Nuclear Factor-kappaB in endothelial cells. Journal of cardiovascular pharmacology 49, 376-383, https://doi.org/10.1097/ FJC.0b013e31804a5e34 (2007).

20. Biedermann, J. S. et al. Rosuvastatin use improves measures of coagulation in patients with venous thrombosis. Eur Heart J 39, 1740-1747, https://doi.org/10.1093/eurheartj/ehy014 (2018).

21. Bertolotto, A. et al. More on the effect of atorvastatin on plasma fibrinogen levels in primary hypercholesterolemia. Atherosclerosis 143, 455-457 (1999).

22. Undas, A., Brummel, K. E., Musial, J., Mann, K. G. \& Szczeklik, A. Simvastatin depresses blood clotting by inhibiting activation of prothrombin, factor V, and factor XIII and by enhancing factor Va inactivation. Circulation 103, 2248-2253 (2001).

\section{Acknowledgements}

We would like to thank all members of the SWITCO65+ study for their contributions. We thank Kali Tal for her editorial contribution.

\section{Author Contributions}

R.K., S.B., O.S., A.L., M.M., N.R. and M.F. were responsible for study design and statistical analyses. R.K., M.F., S.B. and N.R. wrote the manuscript. D.A., M.F., N.R., S.B., M.M., O.S., A.L. and J.H.B. critically reviewed and revised the manuscript. All authors approved the final version to be published.

\section{Additional Information}

Competing Interests: The authors declare no competing interests.

Publisher's note Springer Nature remains neutral with regard to jurisdictional claims in published maps and institutional affiliations.

(c) (i) Open Access This article is licensed under a Creative Commons Attribution 4.0 International License, which permits use, sharing, adaptation, distribution and reproduction in any medium or format, as long as you give appropriate credit to the original author(s) and the source, provide a link to the Creative Commons license, and indicate if changes were made. The images or other third party material in this article are included in the article's Creative Commons license, unless indicated otherwise in a credit line to the material. If material is not included in the article's Creative Commons license and your intended use is not permitted by statutory regulation or exceeds the permitted use, you will need to obtain permission directly from the copyright holder. To view a copy of this license, visit http://creativecommons.org/licenses/by/4.0/.

(c) The Author(s) 2019 